\title{
Surfactant-assisted synthesis of titanium nanoMOFs for thin film fabrication
}

Received 00th January 20xx, Accepted 00th January 20xx

\author{
María Romero-Angel, ${ }^{a}$ Javier Castells-Gil,, Víctor Rubio-Giménez, ${ }^{\mathrm{b}}$ Rob Ameloot, ${ }^{\mathrm{b}}$ Sergio Tatay ${ }^{* a}$ \\ and Carlos Martí-Gastaldo*a
}

DOI: $10.1039 / \times 0 \times x 00000 x$

\begin{abstract}
We use dodecanoic acid as a modulator to yield titanium MOF nanoparticles with good control of size and colloid stability and minimum impact to the properties of the framework to enable direct fabrication of crystalline, porous thin films.
\end{abstract}

Metal-Organic Frameworks (MOFs) are coordination polymers built from the assembly of organic and inorganic building blocks into porous architectures. MOFs display unlimited chemical compositions and structures, sizeable porosities and modulable functions for a highly versatile family of molecular materials. The reduction of their particle size to a nanometric regime can result not only in the retention of these properties but also in new features not present in the bulk. MOF nanoparticles or nanoMOFs can display accelerated adsorption/desorption kinetics $^{1}$, superior catalytic activity ${ }^{2}$, enhanced gas selectivity when used in mixed matrix membranes, ${ }^{3}$ or superior properties when used as drug delivery or theranostic agents. ${ }^{4}$ Compared to the synthesis of inorganic nanoparticles (NPs) that is now well established, methods for producing MOF NPs with controllable particle size and surface chemistry are still at an early stage. ${ }^{5}$ It is highly desirable to identify suitable conditions that allow for reproducibly synthesising nanoMOFs and better understanding of the mechanisms that govern their growth. ${ }^{6-8}$

The synthesis of MOF NPs involves a strict control of the nucleation and growth of the framework by optimization of experimental variables such as the concentration of the precursors and the reaction temperature or time. Overall, NPs can be produced by using different methods like rapid nucleation, nanoreactor confinement, surfactant-assisted synthesis and coordination modulation (CMs), or a combination of them. The use of modulators is arguably the dominant choice as they can control self-assembly by coordinating to the metal sites in the edges of the crystal to terminate its growth thus regulating particle size and/or shape. ${ }^{9}$ The CM approach has been predominantly used to control the size, shape and surface chemistry of $\mathrm{Zr}(\mathrm{IV})$-nanoMOFs for control of their porosity ${ }^{10}$, colloid stability ${ }^{11}$, cellular uptake ${ }^{12}$ or processability ${ }^{13}$.

\footnotetext{
a. Instituto de Ciencia Molecular (ICMol). Universitat de València, Catedrático José Beltrán-2, 46980, Paterna (Spain)

b. Centre for Membrane Separations, Adsorption, Catalysis and Spectroscopy for Sustainable Solutions (cMACS), KU Leuven, Celestijnenlaan 200F, 3001 Leuven (Belgium)

† Electronic Supplementary Information (ESI) available: Experimental details; materials and reagents, synthesis of the NPs, PXRD, profile fitting, SEM, ${ }^{1} \mathrm{H}-\mathrm{NMR}, \mathrm{N}_{2}$ isotherms, DLS, TEM, UV-Vis of the NPs and AFM and Kr isotherms of the film.
}

However, the application of modulation to Ti(IV) frameworks remains comparably underexplored. This is possibly due to the more limited pool of MOFs available and the complex hydrolysis equilibrium of this metal in solution. The intrinsic properties of titanium such as its high polarizing power, low-toxicity, natural abundance or photo-redox activity endow these frameworks with desirable chemical stability or photocatalytic activity ${ }^{14}$. Provided good control of size and porosity, the synthesis of $\mathrm{Ti}$ nanoMOFs would be desirable for the development of biomedical or photocatalytic applications. Previous works demonstrate the difficulties in synthesising homogeneous dispersions of NPs of the archetypical MIL-125- $\mathrm{NH}_{2}$ below 200 $\mathrm{nm} \cdot{ }^{15-17}$ To the best of our knowledge, there are only two reports of nanocrystals close to the $100 \mathrm{~nm}$ nanoparticle limit by using either a polymer as directing agent ${ }^{18}$ or $p$-toluylic acid as modulator. ${ }^{19}$ As result of polymer encapsulation or modulator incorporation, these two routes impose significant changes to the local structure of the framework by introduction of defects or its porosity compared to bulk crystals. We argued the use of a fatty carboxylic acid as surfactant might be more effective in yielding nanocrystals with minimum impact to the properties of the bulk due to ineffective loading or incorporation to the structure of the framework. Our study shows the ability of dodecanoic acid (DA) to control the synthesis of Ti-nanoMOFs for producing homogeneous dispersions of non-defective NPs down to $70 \mathrm{~nm}$ in size, that show excellent colloid stability, and can be assembled into highquality thin films that retain the porosity of the bulk.

We followed a systematic approach to accelerate the exploration of the chemical space and to define the optimal conditions for controlling the formation of NPs. We run 18 reactions that encompassed changes to ligand:metal (L:M) and modulator:metal (Mod:M) molar ratios. The use of a synthesis robot allowed for a quick and automated exploration of the different reaction conditions whilst ensuring maximum reproducibility. ${ }^{20,21}$ In addition to the conditions generally accepted (L:M 1.5 and Mod:M 0), ${ }^{10}$ we also included L:M ratios of 0.5 and 1.0 and Mod:M ratios of 0 to 50 to our synthetic sets. All samples were prepared by solvothermal reaction of 2aminoterephtalic acid, Ti(IV) tetraisopropoxide (TTIP) and DA as modulator in a 9:1 mixture of $\mathrm{N}, \mathrm{N}$-dimethyl formamide (DMF) and methanol (MeOH) at $120^{\circ} \mathrm{C}$. Specific variations to L:M and Mod:M molar ratios are indicated in Table $\mathbf{S 1}$. The manipulation of all the reagents was carried under inert atmosphere to avoid the formation of amorphous $\mathrm{TiO}_{2}$, which might result from the 

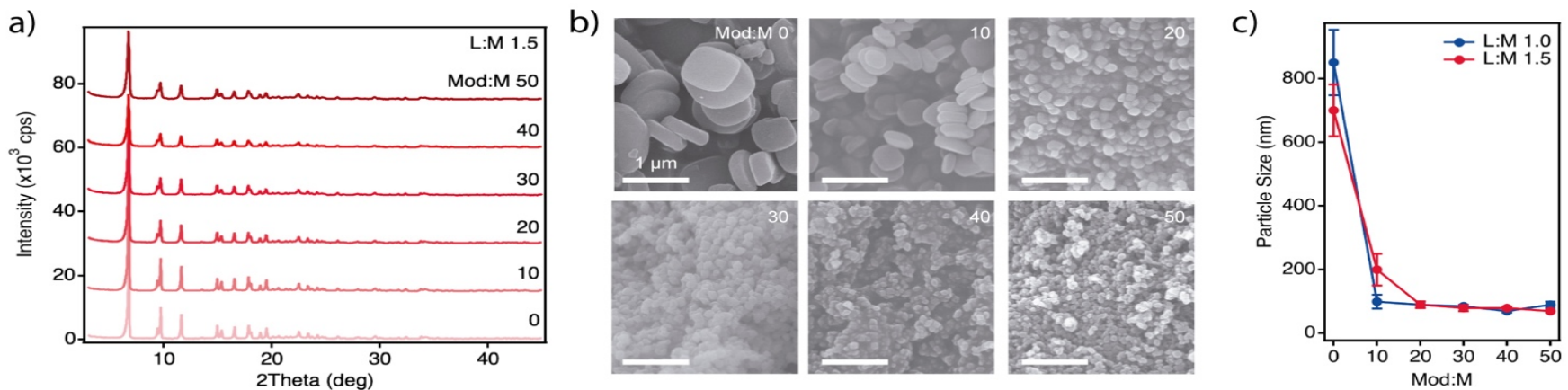

Figure 1. a) PXRD and b) SEM images of the MIL-125- $\mathrm{NH}_{2}$ samples obtained for increasing concentration of DA and a linker-to-metal molar ration fixed to 1.5, c) Variation of particle size with the L:M ratio as determined from SEM (square) or Scherrer analysis of the PXRD patterns (circles).

hydrolysis of the titanium precursor with the ambient moisture. Products were synthesized in $25 \mathrm{~mL}$ Schott bottles, and could be easily scaled-up by 4 -fold in $100 \mathrm{~mL}$ reactors to yield yellow polycrystalline solids in all cases.

The crystallinity of the samples was analysed with powder X-ray diffraction (PXRD). As shown in Figure 1a and Figures S1-S2, crystalline samples were only obtained for L:M ratios above 0.5 , regardless of the amount of modulator added. This suggests that the linker plays a critical role in controlling the crystallization of the framework, imposing the use of a stoichiometric excess even in the presence of DA. The set of amorphous samples for $\mathrm{L}: \mathrm{M}=0.5$ was discarded and will not be further considered along the text. The unit cell parameters of all crystalline powders were determined by Lebail refinement of the corresponding PXRD patterns (Figure S3). All are in excellent agreement with the crystallographic parameters of pristine MIL$125^{22}$ and showed marginal variations along the series (Table S2). Next, scanning electron microscopy (SEM) was used to study the particle size and morphology of the isolated solids (Figure 1b and Figure S4). The influence of the L:M ratio in the particle size becomes negligible when an excess of modulator is used as confirmed by the overlap of both sets for Mod:M ratios above 20 (Figure 1c). This change in particle size is in good agreement with the trends determined from PXRD by using the Scherrer equation (Table S3). We also infer a change in morphology from plate-like particles with rounded corners to truncated octahedra, that is progressively observed along with the increase of modulator concentration. Similar trends in particle morphology can be prompted by varying the concentration of reactants ${ }^{23}$ or by using directing agents, i.e. surfactants. ${ }^{24,19}$ The change in size observed with the addition of DA is also consistent with the effect of other directing agents $^{19}$ and modulators ${ }^{18}$ over the crystal growth of MIL-125$\mathrm{NH}_{2}$.

${ }^{1} \mathrm{H}-\mathrm{NMR}$ experiments of washed samples were used to quantify the amount of DA acid incorporated to the framework. Digestion of the solids in a $\mathrm{D}_{2} \mathrm{SO}_{4}$ : d6-DMSO confirmed that the concentration of DA in the structure is negligible (Figure S5). $\mathrm{N}_{2}$ sorption measurements at $77 \mathrm{~K}$ of the L:M 1.5 and 1.0 sets of solids (Figure 2a and Figure S6a) showed a type-I isotherm characteristic of microporous materials with BET surface areas and micropore volumes that display small changes with respect to the bulk crystals prepared in absence of DA. Changes are comparatively smaller for the L:M 1.5 samples, with deviations of near $10 \%$, compared to an increase of $c a$. $20 \%$ for the L:M 1.0 set (Table S4). We also observed a progressive increase in the uptake at $P / P_{0}>0.9$ with increasing amounts of $D A$, that is characteristic of interparticle mesoporosity associated to the reduction of the particle size. Only the Mod:M=50 samples of both sets show drastic differences in their textural properties indicative of significant changes to their microstructure. Except for these samples, the isotherm shape and accessible porosity remain insensitive to the surfactant concentration, suggesting that DA molecules are not incorporated to the framework during the synthesis. This is further confirmed by the pore size distributions (PSDs) calculated by non-linear DFT methods for both series, which show no changes in the pore width regardless of DA concentration (Figure $\mathbf{2} \mathbf{b}$ and Figure $\mathbf{S 6} \mathbf{b}$ ). This behaviour differs from previous examples, for which size modulation leads to changes in the porosity of MIL-125- $\mathrm{NH}_{2}$ nanoparticles by the introduction of defects ${ }^{18}$ or pore blocking ${ }^{19}$. Even though DA is a C12 fatty acid of amphiphilic nature, more prone to induce the formation of micelles and can behave as a templating agent during MOF formation, ${ }^{25}$ our results confirm that the addition of DA can be also effective in controlling particle size with a minimum impact to the porosity or structure of the framework.

From this preliminary analysis, we shifted focus to the L:M 1.5 Mod:M 30 sample, for a more detailed study representative of MIL-125- $\mathrm{NH}_{2}$ NPs respectful with the properties of the bulk (B). The thermogravimetric analysis (TGA) of both samples in air reveal similar profiles, with a first weight loss at around $60^{\circ} \mathrm{C}$ which is attributed to the removal of methanol in the pores. This is followed by a plateau up to the final weight loss at $c a .320^{\circ} \mathrm{C}$, which corresponds to the gradual decomposition of the framework to form $\mathrm{TiO}_{2}$ (Figure 2c). The thermal decomposition profile along with ${ }^{1} \mathrm{H}-\mathrm{NMR}, \mathrm{BET}$ and PSD further supports that DA modulation leads exclusively to particle size reduction with negligible changes to the thermal stability or structure of MIL125- $\mathrm{NH}_{2}$. Fourier Transformed Infrared spectroscopy (FTIR) measurements of the NPs discarded the presence of free carboxylic groups at $1659 \mathrm{~cm}^{-1}$ or vibrational $\mathrm{Csp}_{3}-\mathrm{H}$ stretching bands of the aliphatic chain at $3000-2800 \mathrm{~cm}^{-1}$ (Figure 2d), which might be indicative of the presence of DA molecules physiosorbed on the surface of the nanocrystals or occluded in 
a)

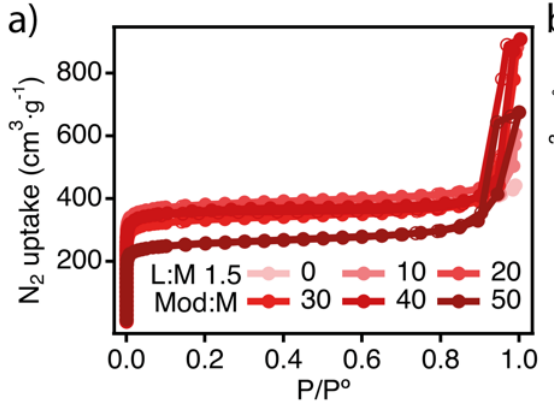

b)

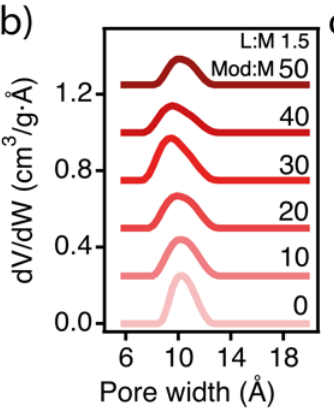

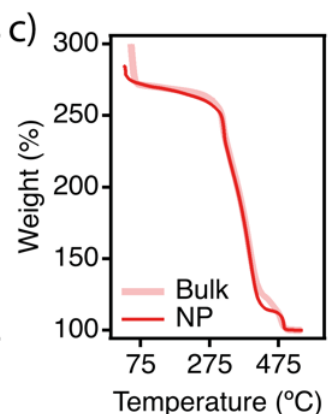

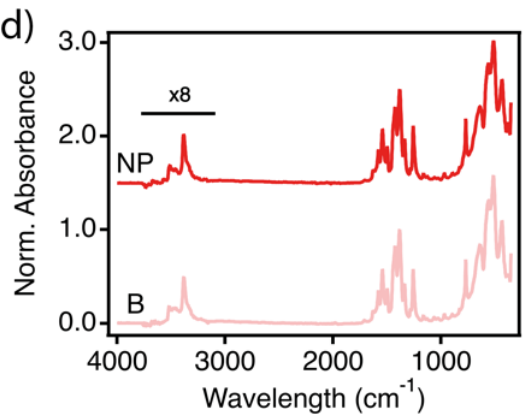

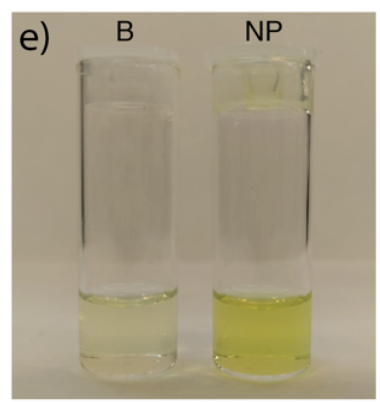
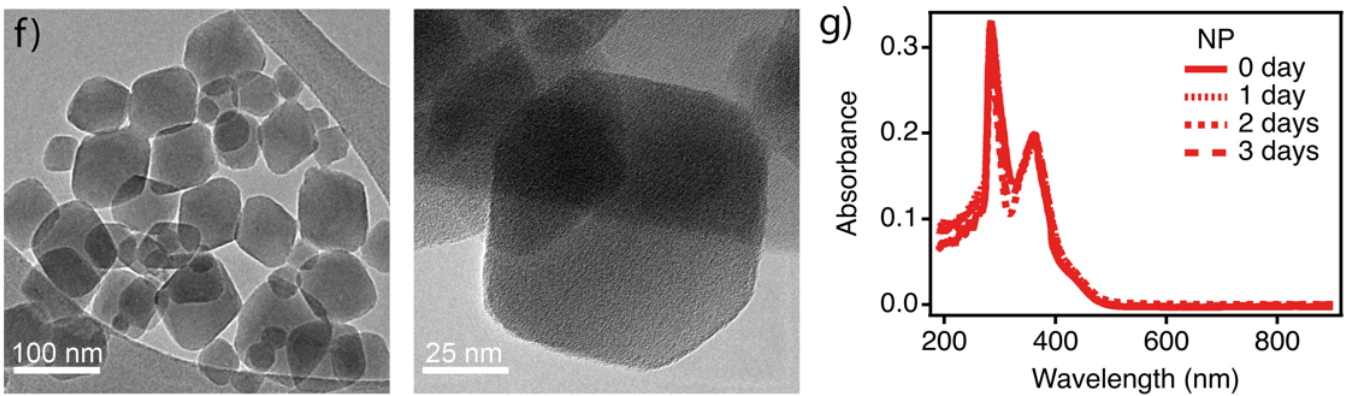

Figure 2. a) $\mathrm{N}_{2}$ adsorption isotherms and b) PSD of the MIL-125- $\mathrm{NH}_{2} \mathrm{~L}: \mathrm{M} 1.5$ samples, C) TGA and d) FTIR of the bulk (B, L:M 1.5, Mod:M 0) and nanoparticulated (NP, L:M 1.5, Mod:M 30) samples, e) optical photograph of B (left) and NP (right) methanol suspensions few minutes after their preparation, f) HRTEM images of the NP sample, g) UV-Vis of the methanol NP dispersion showed in e) after 24 to 72 hours.

the pores of framework. Next, we evaluated the changes in colloidal stability for dispersions of the bulk and the NPs in different solvents. $20 \mathrm{mg}$ of each were dispersed in $10 \mathrm{~mL}$ of water, toluene, chloroform, dimethylformamide (DMF), dichloromethane (DCM), methanol and ethanol, and sonicated for 1 hour. After 24 hours only the NP suspensions in methanol showed good stability whereas bulk samples rapidly sedimented at the bottom of the vial in all cases (Figure 2e). Dynamic light scattering (DLS) measurements on NPs yielded hydrodynamic particle size of $70 \pm 5$, with polydispersity index of $0.06 \pm 0.03$ when suspended in methanol (Figure S7). High resolution transmission electron microscopy (HR-TEM) images were taken from a methanol dispersion. Figure $\mathbf{2 f}$ and Figure $\mathbf{S 8}$ show the presence of truncated octahedra with a narrow size distribution and an average particle size of $90 \pm 22 \mathrm{~nm}$, in close agreement with the particle size anticipated by SEM, XRD and DLS data. As for the colloid stability, UV-Vis studies confirmed that the suspensions are stable for more than 2 days (Figure $\mathbf{2 g}$ and Figure S10), with methanol showing slightly better stability.

One of the challenges for the integration of MOFs into electronic, electrochemical or optoelectronic devices is their processing as homogeneous films. ${ }^{26-29}$ We argued the narrow size dispersion and good colloid stability of the NPs prepared would be ideal to facilitate their processing. Accordingly, we studied three thin film preparation techniques: dip coating, spray coating and spin coating (see Section S11). As a solid support we chose indium-titanium oxide (ITO), a transparent conducting oxide of broad use in research and industry. Although NPs can be successfully deposited on ITO in all cases, the most homogeneous films were always obtained by using spin coating (Figure S13). SEM and AFM images of the film show a homogeneous distribution of the NPs throughout the substrate (Figure 3a). This corresponds to a fairly compact, flat arrangement of the NPs according to the AFM topographic analysis of the film (Figure 3b). A film thickness of $104 \pm 17 \mathrm{~nm}$
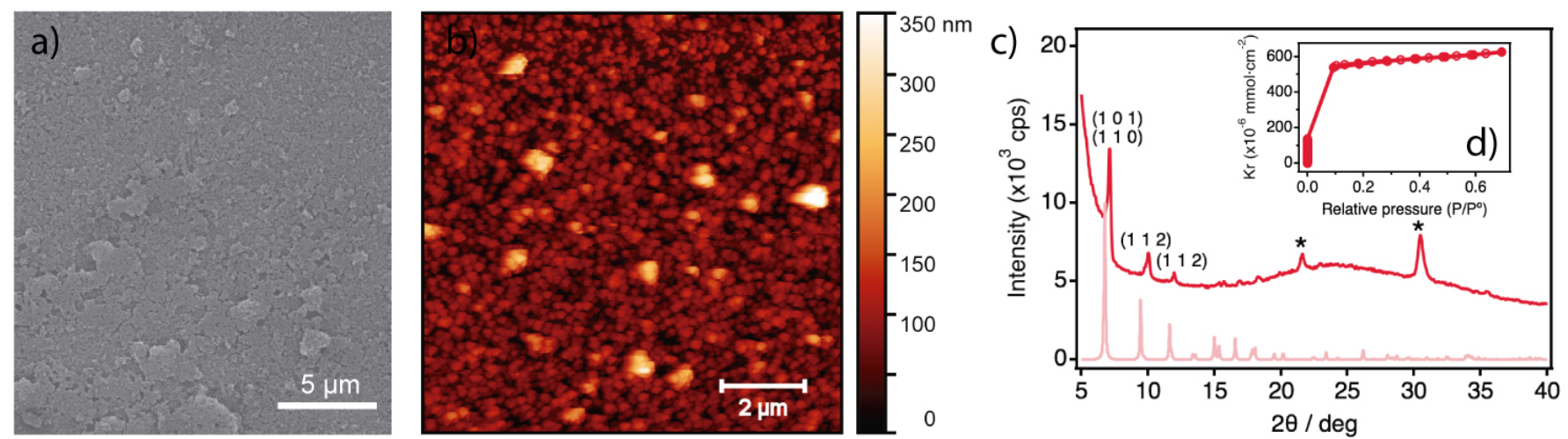

Figure 3. a) SEM and b) AFM images of films fabricated by spin coating, c) Gonio-mode XRD of the film (red) and simulated diffractogram (pink), ITO substrate peaks are marked with ${ }^{*}$, d) Inset: Kr adsorption isotherm of the film. 
was determined by AFM after scratching the film with a soft pointy tool. Considering the size determined with HR-TEM, this value suggests the deposition of roughly one NP layer. ${ }^{30}$ Furthermore, XRD shows the characteristic Bragg peaks of the MIL-125 structure with no significant change in their relative intensities (Figure 3c), thus confirming the crystallinity of the films and ruling out any preferential orientation of the NPs on the substrate. Finally, we measured the porosity of the film by means of $\mathrm{Kr}$ physisorption (Figure 3c, Figure S15), a very sensitive method to assess the porosity of thin films with small absolute surface areas. ${ }^{31}$ The porosimetry data confirm the effective transfer of the accessible porosity of bulk MIL-125- $\mathrm{NH}_{2}$ to the film, which displays a calculated volumetric BET surface area of ca. $1700 \mathrm{~m}^{2} \cdot \mathrm{cm}^{-3}$. This is slightly above the $\mathrm{Kr}$ surface area calculated via Monte Carlo sampling (1610.1 $\left.\mathrm{m}^{2} \cdot \mathrm{cm}^{-3}\right)$ for MIL-125- $\mathrm{NH}_{2}$.

In summary, we have demonstrated the ability of DA to regulate the formation of titanium nanoMOFs with good control over their size and colloid stability. Compared to other methods, the use of a surfactant combines control of the assembly of the framework with negligible incorporation of the modulator for minimum impact on its structure or porosity. This allows for easy fabrication of crystalline thin films that retain the porosity of the bulk simply by spin coating.

We thank the European Union (ERC Stg Chem-fs-MOF 714122 and VAPORE 716472), the Spanish government (CEX2019000919-M, RTI2018-098568-A-I00, CTQ2017-83486-P \& RYC2016-1981) and Research Foundation Flanders (FWO Vlaanderen; G083016N, G85720N and 12Z6520N) for funding. We also thank the SCSIE of the University of València for access to research facilities.

\section{Notes and references}

Y. Sakata, S. Furukawa, M. Kondo, K. Hirai, N. Horike, Y. Takashima H. Uehara, N. Louvain, M. Meilikhov, T. Tsuruoka, S. Isoda, W. Kosaka, O. Sakata and S. Kitagawa, Science (80-. )., 2013, 339, 193196. Wang and D. Astruc, Chem. Rev., 2020, 120, 1438-1511. L. Xiang, L. Sheng, C. Wang, L. Zhang, Y. Pan and Y. Li, Adv. Mater., 2017, 29, 1606999.

Mater., 2018, 30, 1707365.

5 S. Wang, C. M. McGuirk, A. d'Aquino, J. A. Mason and C. A. Mirkin, Adv. Mater., 2018, 30, 1800202.

6 F. Haase, P. Hirschle, R. Freund, S. Furukawa, Z. Ji and S. Wuttke, Angew. Chemie - Int. Ed., 2020, 59, 22350-22370.

7 C. R. Marshall, E. E. Timmel, S. A. Staudhammer and C. K. Brozek, Chem. Sci., 2020, 11, 11539-11547.

8 C. R. Marshall, S. A. Staudhammer and C. K. Brozek, Chem. Sci., 2019, 10, 9396-9408.

9 R. S. Forgan, Chem. Sci., 2020, 11, 4546-4562.

10 G. Cai and H. L. Jiang, Angew. Chemie - Int. Ed., 2017, 56, 563-567.

11 S. Wang, C. M. McGuirk, M. B. Ross, S. Wang, P. Chen, H. Xing, Y. Liu and C. A. Mirkin, J. Am. Chem. Soc., 2017, 139, 9827-9830.

I. Abánades Lázaro, S. Haddad, S. Sacca, C. Orellana-Tavra, D. Fairen-Jimenez and R. S. Forgan, Chem, 2017, 2, 561-578.

13 B. Bueken, N. Van Velthoven, T. Willhammar, T. Stassin, I. Stassen, D. A. Keen, G. V. Baron, J. F. M. Denayer, R. Ameloot, S. Bals, D. De Vos and T. D. Bennett, Chem. Sci., 2017, 8, 3939-3948.
H. Assi, G. Mouchaham, N. Steunou, T. Devic and C. Serre, Chem. Soc. Rev., 2017, 46, 3431-3452.

S. Rojas, N. Guillou and P. Horcajada, ACS Appl. Mater. Interfaces, , DOI:10.1021/acsami.9b06472.

F. Guo, J. H. Guo, P. Wang, Y. S. Kang, Y. Liu, J. Zhao and W. Y. Sun, Chem. Sci., 2019, 10, 4834-4838.

Z. Su, J. H. Chen, X. Sun, Y. Huang and X. Dong, RSC Adv., 2015, 5, 99008-99017.

S. Hu, M. Liu, X. Guo, K. Li, Y. Han, C. Song and G. Zhang, Cryst. Growth Des., 2017, 17, 6586-6595.

D. K. Roh, H. Jae, H. Mun, J. H. Jo and W. S. Chi, Mater. Sci. Eng. B Solid-State Mater. Adv. Technol., 2021, 263, 114833.

N. M. Padial, J. Castells-Gil, N. Almora-Barrios, M. Romero-Angel, I. Da Silva, M. Barawi, A. García-Sánchez, V. A. De La Peña O’Shea and C. Martí-Gastaldo, J. Am. Chem. Soc., 2019, 141, 1312413133.

J. Castells-Gil, N. M. Padial, N. Almora-Barrios, I. Da Silva, D. Mateo, J. Albero, H. García and C. Martí-Gastaldo, Chem. Sci. 2019, 10, 4313-4321.

M. Dan-Hardi, C. Serre, T. Frot, L. Rozes, G. Maurin, C. Sanchez and G. Férey, J. Am. Chem. Soc., 2009, 131, 10857-10859.

S. Hu, M. Liu, K. Li, Y. Zuo, A. Zhang, C. Song, G. Zhang and X. Guo, CrystEngComm, 2014, 16, 9645-9650.

F. Guo, J. H. Guo, P. Wang, Y. S. Kang, Y. Liu, J. Zhao and W. Y. Sun, Chem. Sci., 2019, 10, 4834-4838.

A. Kirchon, J. Li, F. Xia, G. S. Day, B. Becker, W. Chen, H. J. Sue, Y. Fang and H. C. Zhou, Angew. Chemie - Int. Ed., 2019, 58, 1242512430.

J. Meng, X. Liu, C. Niu, Q. Pang, J. Li, F. Liu, Z. Liu and L. Mai, Chem. Soc. Rev., 2020, 49, 3142-3186.

J. Liu and C. Wöll, Chem. Soc. Rev., 2017, 46, 5730-5770.

I. Stassen, N. Burtch, A. Talin, P. Falcaro, M. Allendorf and R. Ameloot, Chem. Soc. Rev., 2017, 46, 3185-3241.

V. Rubio-Giménez, S. Tatay and C. Martí-Gastaldo, Chem. Soc. Rev., 2020, 49, 5601-5638.

S. Tatay, M. Galbiati, S. Delprat, C. Barraud, K. Bouzehouane, S. Collin, C. Deranlot, E. Jacquet, P. Seneor, R. Mattana and F. Petroff, J. Phys. Condens. Matter, 2016, 28, 094010. T. Stassin, R. Verbeke, A. J. Cruz, S. Rodríguez-Hermida, I. Stassen, J. Marreiros, M. Krishtab, M. Dickmann, W. Egger, I. F. J. Vankelecom, S. Furukawa, D. De Vos, D. Grosso, M. Thommes and R. Ameloot, Adv. Mater., 2021, 33, 2170133. 